\title{
Tabellen
}

\section{Verzeichnis der Tabellen}

Tabelle 1 Liste der Londoner Theater (1800 bis 1930)

Tabelle 2 Liste der Berliner Theater (1800 bis 1930)

Tabelle 3 Liste ausgewählter Musical Comedies

Tabelle 4 Liste ausgewählter Revuen (London)

Tabelle 5 Liste ausgewählter in Berlin uraufgeführter Operetten

Tabelle 6 Liste ausgewählter Berliner Revuen

Tabelle 7 Transfer London - Berlin

Tabelle 8 Transfer Berlin - London

Tabelle 9 Anzahl der in London genehmigten und verbotenen Stücke

Tabelle 10 Anzahl der in Berlin genehmigten und verbotenen Stücke

Tabelle 11 Schauspielerinnen und Schauspieler in London/Großbritannien

Tabelle 12 Angestellte im Bereich Musik und Theater in Berlin/Deutschland 
Tabelle 1: Londoner Theater

\begin{tabular}{|c|c|c|c|c|}
\hline Theater & Ort & Eröffnet & Geschlossen & Plätze \\
\hline \multicolumn{5}{|l|}{ 1. West End } \\
\hline Adelphi Theatre & Westminster & 1806 & & 1500 \\
\hline Aldwych Theatre & Westminster & 1905 & & 1100 \\
\hline Ambassadors Theatre & Westminster & 1913 & & 490 \\
\hline Apollo Theatre & Westminster & 1901 & & 893 \\
\hline Carlton Theatre & Westminster & 1927 & 1929 & 1150 \\
\hline Comedy Theatre & Westminster & 1881 & & 1055 \\
\hline Covent Garden & Westminster & 1732 & & 2299 \\
\hline Criterion Theatre, & Westminster & 1874 & & 560 \\
\hline Daly's Theatre & Westminster & 1893 & 1938 & 1200 \\
\hline Dominion Theatre & Westminster & 1929 & 1932 & 2800 \\
\hline Drury Lane & Westminster & 1663 & & 3060 \\
\hline Duchess Theatre & Westminster & 1929 & & 491 \\
\hline Duke of York's Theatre & Westminster & 1892 & & 900 \\
\hline Fortune Theatre & Westminster & 1924 & & 473 \\
\hline Gaiety Theatre & Westminster & 1868 & 1903 & 1126 \\
\hline Gaiety Theatre & Westminster & 1903 & 1939 & 1267 \\
\hline Garrick & Westminster & 1889 & & 773 \\
\hline Globe Theatre & Westminster & 1868 & 1902 & 1000 \\
\hline Haymarket Theatre Royal & Westminster & 1720 & & 1159 \\
\hline Her Majesty's Theatre & Westminster & 1705 & & 1319 \\
\hline Hicks Theatre & Westminster & 1906 & & 889 \\
\hline Holborn Theatre & Holborn & 1867 & 1887 & \\
\hline Holborn Theatre Royal & Holborn & 1866 & 1880 & 1500 \\
\hline Imperial Theatre, The & Westminster & 1876 & 1907 & 1293 \\
\hline Kingsway Theatre, The & Holborn & 1882 & 1941 & 650 \\
\hline Leicester Square Theatre & Westminster & 1930 & & 2000 \\
\hline Little Theatre, The & Westminster & 1910 & 1941 & 309 \\
\hline London Coliseum & Westminster & 1904 & & 3389 \\
\hline London Hippodrome & Westminster & 1900 & & 1340 \\
\hline London Palladium & Westminster & 1910 & & 1090 \\
\hline London Pavilion & Westminster & 1885 & 1934 & \\
\hline Lyceum & Westminster & 1765 & & 2100 \\
\hline Lyric Theatre & Westminster & 1888 & & 1306 \\
\hline New Theatre & Westminster & 1903 & & 938 \\
\hline Olympic Theatre & Westminster & 1806 & 1899 & 2150 \\
\hline Opera Comique & Westminster & 1870 & 1899 & 862 \\
\hline Palace Theatre & Westminster & 1891 & & 1697 \\
\hline Piccadilly Theatre & Westminster & 1928 & & 1395 \\
\hline Playhouse & Westminster & 1882 & & 1500 \\
\hline Prince Edward Theatre & Westminster & 1930 & & 1800 \\
\hline Prince of Wales Theatre & Westminster & 1884 & & 960 \\
\hline Prince's Theatre & Holborn & 1911 & & 2392 \\
\hline Princess's Theatre & Westminster & 1840 & 1902 & 1750 \\
\hline Queen's Theatre & Westminster & 1867 & 1879 & 4000 \\
\hline Queen's Theatre & Westminster & 1907 & & 1160 \\
\hline Royal Strand Theatre & Westminster & 1831 & 1905 & 1500 \\
\hline
\end{tabular}




\begin{tabular}{|c|c|c|c|c|}
\hline Theater & Ort & Eröffnet & Geschlossen & Plätze \\
\hline Royalty Theatre & Westminster & 1850 & 1938 & 657 \\
\hline Saville Theatre & Westminster & 1931 & & 1426 \\
\hline Savoy Theatre & Westminster & 1881 & & 1300 \\
\hline Shaftesbury Theatre & Holborn & 1888 & 1941 & 1196 \\
\hline Strand Theatre (1) & Westminster & 1832 & 1905 & 1500 \\
\hline Strand Theatre (2) & Westminster & 1905 & & 1193 \\
\hline Terry's Theatre & Westminster & 1887 & 1910 & 888 \\
\hline Toole's Theatre & Westminster & 1855 & 1895 & 600 \\
\hline Vaudeville Theatre & Westminster & 1870 & & 1000 \\
\hline Victoria Palace & Victoria & $1863 / 1911$ & & $2440 / 1500$ \\
\hline Westminster Theatre & Westminster & 1931 & & 650 \\
\hline Whitehall Theatre & Westminster & 1930 & & 620 \\
\hline Windmill Theatre & Westminster & 1931 & & 320 \\
\hline Winter Garden Theatre & Holborn & 1911 & & 1800 \\
\hline Wyndham's Theatre & Westminster & 1899 & & 1200 \\
\hline \multicolumn{5}{|l|}{ 2. East End } \\
\hline Alexandra Theatre & Islington & 1740 & 1871 & 1900 \\
\hline Britannia & Hoxton & 1841 & 1923 & 2972 \\
\hline City of London & Bishopsgate & 1835 & 1871 & 2500 \\
\hline East London & Stepney & 1834 & 1940 & 2150 \\
\hline Old Vic Theatre & Lambeth & 1818 & & 1454 \\
\hline Pavilion Theatre & Stepney & 1828 & 1934 & 3500 \\
\hline Queen's Theatre & Poplar & 1856 & 1958 & 1360 \\
\hline Sadler's Wells & Islington & 1683 & & 1600 \\
\hline St. George's Hall & Westminster & 1867 & 1941 & 1000 \\
\hline St. James's & Westminster & 1835 & 1957 & 1200 \\
\hline St. Martin's Theatre & Holborn & 1916 & & 600 \\
\hline \multicolumn{5}{|l|}{ 2. Vorstädte } \\
\hline Alexandra Theatre & Stoke Newington & 1897 & 1950 & 3000 \\
\hline Astley's Amphitheatre & Lambeth & 1773 & 1893 & 2407 \\
\hline Balham Hippodrome & Balham & 1899 & 1939 & 2500 \\
\hline Broadway Theatre & New Cross & 1867 & 1911 & 1372 \\
\hline Camden Theatre & Camden & 1901 & 1924 & 2434 \\
\hline Coronet Theatre & Islington & 1898 & 1923 & 1143 \\
\hline Dalston Theatre & Hackney & 1886 & 1923 & 1030 \\
\hline Elephant and Castle & Southwark & 1872 & 1928 & 2203 \\
\hline Embassy Theatre & Hampstead & 1927 & & 678 \\
\hline Grand Theatre & Fulham & 1897 & 1912 & 2239 \\
\hline Grecian Theatre & Shoreditch & 1825 & 1882 & 1850 \\
\hline Greenwich Theatre & Greenwich & 1864 & 1910 & 721 \\
\hline Kennington Theatre & Kennington & 1898 & 1921 & 1347 \\
\hline Lyric Opera House & Hammersmith & 1888 & & 800 \\
\hline Marlborough Theatre & Islington & 1903 & 1919 & 2612 \\
\hline Park Theatre & Camden & 1873 & 1881 & \\
\hline Parkhurst Theatre & Islington & 1890 & 1909 & 600 \\
\hline Peckham Hippodrome & Peckham & 1898 & 1912 & 2600 \\
\hline
\end{tabular}




\begin{tabular}{lllll}
\hline Theater & Ort & Eröffnet & Geschlossen Plätze \\
\hline Phoenix Theatre & Camden & 1930 & & 1011 \\
Royal Artillery Theatre & Woolwich & 1905 & & 1000 \\
Royal County Theatre & Kingston & 1897 & 1912 & 1300 \\
Royal Court Theatre & Chelsea & 1888 & & 841 \\
Scala Theatre & St. Pancras & 1772 & 1969 & 600 \\
Shakespeare Theatre & Battersea & 1896 & 1923 & 1205 \\
Standard Theatre & Shoreditch & 1837 & 1926 & 3000 \\
Surrey & Lambeth & 1782 & 1920 & 2161 \\
Terriss & Rotherhithe & 1899 & 1927 & 2087 \\
Theatre Royal Stratford & Stratford & 1884 & & 460 \\
\hline
\end{tabular}

Quelle: Era Almanack; Green Room Book; Who's Who; Mander und Mitchenson, The Theatres of London; dies., Lost Theatres of London; HOWARD, London Theatres and Music Halls; EARL und SeLL, The Theatres Trust Guide to British Theatres. 
Tabelle 2: Berliner Theater

\begin{tabular}{|c|c|c|c|c|c|}
\hline Name & Alternative Name & Ort & Eröffnet & Geschlossen & Sitzplätze \\
\hline $\begin{array}{l}\text { Königliches } \\
\text { Opernhaus }\end{array}$ & $\begin{array}{l}\text { Opernhaus Unter den Linden, } \\
\text { Staatsoper Unter den Linden }\end{array}$ & $\begin{array}{l}\text { Gendarmen- } \\
\text { markt }\end{array}$ & 1742 & & 1831 \\
\hline $\begin{array}{l}\text { Königliches } \\
\text { Schauspiel- } \\
\text { haus }\end{array}$ & $\begin{array}{l}\text { Königliches Nationaltheater, } \\
\text { Staatliches Schauspielhaus }\end{array}$ & $\begin{array}{l}\text { Gendarmen- } \\
\text { markt }\end{array}$ & 1776 & & 1005 \\
\hline $\begin{array}{l}\text { Königstädti- } \\
\text { sches Theater }\end{array}$ & $\begin{array}{l}\text { Wallner-Theater, Bundes- } \\
\text { hallen-Theater, Heinsdorff- } \\
\text { Theater, Alhambra-Theater, } \\
\text { Königstädtisches Theater, } \\
\text { Berliner Stadttheater }\end{array}$ & $\begin{array}{l}\text { Alexander- } \\
\text { platz }\end{array}$ & 1824 & 1851 & \\
\hline $\begin{array}{l}\text { Neues König- } \\
\text { liches Opern- } \\
\text { haus }\end{array}$ & $\begin{array}{l}\text { Krolls Etablissement (1844), } \\
\text { Krollsches Theater (1874), } \\
\text { Neues Königliches Theater } \\
\text { (1895), Staatsoper am Platz der } \\
\text { Republik (1921) }\end{array}$ & Tiergarten & 1844 & 1945 & 2041 \\
\hline $\begin{array}{l}\text { Schiller- } \\
\text { Theater } \\
\text { (Nord) }\end{array}$ & $\begin{array}{l}\text { Callenbachs Sommertheater, } \\
\text { Woltersdorff-Theater, Neues } \\
\text { Friedrich-Wilhelm-Theater }\end{array}$ & $\begin{array}{l}\text { Chausee- } \\
\text { straße }\end{array}$ & 1848 & 1925 & 1500 \\
\hline $\begin{array}{l}\text { Deutsches } \\
\text { Theater }\end{array}$ & $\begin{array}{l}\text { Friedrich-Wilhelmstädtisches } \\
\text { Theater (bis 1883) }\end{array}$ & $\begin{array}{l}\text { Schumann- } \\
\text { straße }\end{array}$ & 1848 & & 1000 \\
\hline $\begin{array}{l}\text { Vorstädtisches } \\
\text { Theater }\end{array}$ & Germania-Theater & $\begin{array}{l}\text { Wein- } \\
\text { bergsweg }\end{array}$ & 1849 & 1882 & \\
\hline $\begin{array}{l}\text { Berliner } \\
\text { Theater }\end{array}$ & $\begin{array}{l}\text { Neues Königsstädtisches } \\
\text { Theater, Walhalla-Theater, } \\
\text { Walhalla-Operetten-Theater }\end{array}$ & $\begin{array}{l}\text { Charlotten- } \\
\text { straße }\end{array}$ & 1850 & 1935 & 1371 \\
\hline $\begin{array}{l}\text { Belle-Alliance- } \\
\text { Theater }\end{array}$ & $\begin{array}{l}\text { Berliner Volksoper (1905- } \\
\text { 1910), Lortzing-Theater }\end{array}$ & $\begin{array}{l}\text { Belle-Alli- } \\
\text { ance-Straße }\end{array}$ & 1853 & 1913 & 1600 \\
\hline $\begin{array}{l}\text { American- } \\
\text { Theater }\end{array}$ & $\begin{array}{l}\text { Königstädtisches Vaudeville- } \\
\text { Theater, Wallner-Theater, Bun- } \\
\text { deshallen-Theater, } \\
\text { Königstädtisches Theater, } \\
\text { Alhambra-Theater, Berliner } \\
\text { Stadttheater }\end{array}$ & $\begin{array}{l}\text { Blumen- } \\
\text { straße }\end{array}$ & 1855 & & \\
\hline $\begin{array}{l}\text { Victoria- } \\
\text { Theater }\end{array}$ & & Münzstraße & 1859 & 189 & \\
\hline $\begin{array}{l}\text { Wallner- } \\
\text { Theater }\end{array}$ & $\begin{array}{l}\text { Schiller-Theater Ost, Wallner- } \\
\text { Theater }\end{array}$ & $\begin{array}{l}\text { Wallner- } \\
\text { theaterstraße }\end{array}$ & 1864 & & 1251 \\
\hline $\begin{array}{l}\text { Central- } \\
\text { Theater }\end{array}$ & $\begin{array}{l}\text { Reunion-Theater, Henne- } \\
\text { Theater, Thomas-Theater }\end{array}$ & $\begin{array}{l}\text { Alte-Jakob- } \\
\text { Straße }\end{array}$ & 1869 & & 1200 \\
\hline $\begin{array}{l}\text { Thalia- } \\
\text { Theater }\end{array}$ & $\begin{array}{l}\text { Louisenstädtisches Theater, } \\
\text { Eden-Theater, Adolf-Ernst- } \\
\text { Theater }\end{array}$ & $\begin{array}{l}\text { Dresdner } \\
\text { Straße }\end{array}$ & 1869 & & 1100 \\
\hline $\begin{array}{l}\text { Tonhallen- } \\
\text { Theater }\end{array}$ & & $\begin{array}{l}\text { Friedrich- } \\
\text { straße } 112\end{array}$ & 1869 & 1874 & \\
\hline $\begin{array}{l}\text { Vaudeville- } \\
\text { Theater }\end{array}$ & Puhlmann's Vaudeville Theater & $\begin{array}{l}\text { Schönhauser } \\
\text { Allee } 148\end{array}$ & 1869 & & \\
\hline
\end{tabular}




\begin{tabular}{|c|c|c|c|c|c|}
\hline Name & Alternative Name & Ort & Eröffnet & Geschlossen & Sitzplätze \\
\hline $\begin{array}{l}\text { Residenz- } \\
\text { Theater }\end{array}$ & Nowack-Theater $(-1871)$ & Blumenstraße & 1869 & & 600 \\
\hline $\begin{array}{l}\text { National- } \\
\text { Theater }\end{array}$ & & $\begin{array}{l}\text { Weinbergs- } \\
\text { weg 19/20 }\end{array}$ & 1870 & 1883 & 1800 \\
\hline $\begin{array}{l}\text { Bellevue- } \\
\text { Theater }\end{array}$ & & & 1872 & & 400 \\
\hline Stadttheater & & Lindenstraße & 1872 & 1879 & \\
\hline $\begin{array}{l}\text { Borussia- } \\
\text { Theater }\end{array}$ & $\begin{array}{l}\text { Germania-Theater, Deutsches } \\
\text { Reichs-Theater }\end{array}$ & $\begin{array}{l}\text { Wrangel- } \\
\text { straße }\end{array}$ & 1872 & & \\
\hline Stadttheater & & Lützowstraße & 1873 & & \\
\hline $\begin{array}{l}\text { Buntes Thea- } \\
\text { ter }\end{array}$ & $\begin{array}{l}\text { Quargs Vaudeville-Theater, } \\
\text { Königstädtisches Theater, } \\
\text { Alexanderplatz-Theater, } \\
\text { National-Theater, Überbrettl }\end{array}$ & $\begin{array}{l}\text { Alexander- } \\
\text { straße }\end{array}$ & 1874 & 1927 & 750 \\
\hline $\begin{array}{l}\text { American- } \\
\text { Theater }\end{array}$ & & $\begin{array}{l}\text { Dresdner } \\
\text { Straße }\end{array}$ & 1875 & & \\
\hline Rose-Theater & $\begin{array}{l}\text { Ostendtheater, Carl-Weiß- } \\
\text { Theater, Rose-Theater }\end{array}$ & $\begin{array}{l}\text { Große Frank- } \\
\text { furter Straße }\end{array}$ & 1877 & 1944 & 1200 \\
\hline $\begin{array}{l}\text { Reichshallen- } \\
\text { Theater }\end{array}$ & & $\begin{array}{l}\text { Leipziger } \\
\text { Straße }\end{array}$ & 1881 & & \\
\hline $\begin{array}{l}\text { Apollo- } \\
\text { Theater }\end{array}$ & Concordia-Theater & $\begin{array}{l}\text { Friedrich- } \\
\text { straße } 218\end{array}$ & 1884 & 1913 & \\
\hline $\begin{array}{l}\text { Casino- } \\
\text { Theater }\end{array}$ & Gebrüder Richters Varieté & $\begin{array}{l}\text { Lothringer } \\
\text { Straße } 37\end{array}$ & 1887 & & 700 \\
\hline $\begin{array}{l}\text { Artushof- } \\
\text { Theater }\end{array}$ & Bürgerliches Schauspielhaus & $\begin{array}{l}\text { Perleberger } \\
\text { Straße }\end{array}$ & 1888 & & 2000 \\
\hline $\begin{array}{l}\text { Lessing- } \\
\text { Theater }\end{array}$ & & $\begin{array}{l}\text { Friedrich- } \\
\text { Karl-Ufer }\end{array}$ & 1888 & 1945 & 1140 \\
\hline $\begin{array}{l}\text { Passage- } \\
\text { Theater }\end{array}$ & Linden-Cabaret & $\begin{array}{l}\text { Friedrichstra- } \\
\text { ße 158-164 }\end{array}$ & 1888 & 1943 & \\
\hline $\begin{array}{l}\text { Parodie- } \\
\text { Theater }\end{array}$ & & $\begin{array}{l}\text { Oranien- } \\
\text { straße }\end{array}$ & 1889 & & \\
\hline Stadttheater & Stadttheater Moabit & Alt-Moabit & 1889 & & 1200 \\
\hline Neues Theater & $\begin{array}{l}\text { Montis Operetten-Theater, } \\
\text { Neues Operettenhaus, Theater } \\
\text { am Schiffbauerdamm }\end{array}$ & $\begin{array}{l}\text { Schiffbauer- } \\
\text { damm }\end{array}$ & 1892 & & 784 \\
\hline $\begin{array}{l}\text { Metropol- } \\
\text { Theater }\end{array}$ & Theater Unter den Linden & Behrenstraße & 1893 & & 1600 \\
\hline $\begin{array}{l}\text { Luisen- } \\
\text { Theater }\end{array}$ & Volkstheater & $\begin{array}{l}\text { Reichenber- } \\
\text { ger-Straße }\end{array}$ & 1896 & $1922 ?$ & 1226 \\
\hline $\begin{array}{l}\text { Theater des } \\
\text { Westens }\end{array}$ & & Kantstraße & 1896 & & 1700 \\
\hline $\begin{array}{l}\text { Kleines } \\
\text { Theater }\end{array}$ & Schall und Raum (bis 1902) & $\begin{array}{l}\text { Unter den } \\
\text { Linden }\end{array}$ & 1901 & & 400 \\
\hline
\end{tabular}




\begin{tabular}{|c|c|c|c|c|c|}
\hline Name & Alternative Name & Ort & Eröffnet & Geschlossen & Sitzplätze \\
\hline $\begin{array}{l}\text { Trianon- } \\
\text { Theater }\end{array}$ & & $\begin{array}{l}\text { Georgen- } \\
\text { straße }\end{array}$ & 1901 & & 550 \\
\hline Lustspielhaus & & $\begin{array}{l}\text { Friedrich- } \\
\text { straße } 236\end{array}$ & 1904 & & 550 \\
\hline $\begin{array}{l}\text { Komische } \\
\text { Oper }\end{array}$ & Deutsches Schauspielhaus & $\begin{array}{l}\text { Friedrich- } \\
\text { straße } 104\end{array}$ & 1905 & & 1200 \\
\hline Scala-Theater & Folies Caprice (1905-1914) & Linienstraße & 1905 & & \\
\hline $\begin{array}{l}\text { Gebrüder } \\
\text { Herrnfeld } \\
\text { Theater }\end{array}$ & $\begin{array}{l}\text { Theater in der Kommandan- } \\
\text { tenstraße (seit 1918), Theater } \\
\text { in der Stadt }\end{array}$ & $\begin{array}{l}\text { Kommandan- } \\
\text { tenstraße }\end{array}$ & 1906 & 1945 & 1150 \\
\hline $\begin{array}{l}\text { Kammerspiele } \\
\text { des Deutsche } \\
\text { Theater }\end{array}$ & & $\begin{array}{l}\text { Schumann- } \\
\text { straße }\end{array}$ & 1906 & & 400 \\
\hline $\begin{array}{l}\text { Theater am } \\
\text { Nollendorf- } \\
\text { platz }\end{array}$ & Neues Schauspielhaus (1912) & $\begin{array}{l}\text { Nollendorf- } \\
\text { platz } 5\end{array}$ & 1906 & & 1106 \\
\hline $\begin{array}{l}\text { Schiller } \\
\text { Theater West }\end{array}$ & & $\begin{array}{l}\text { Grolman- } \\
\text { straße }\end{array}$ & 1907 & & \\
\hline $\begin{array}{l}\text { Hebbel- } \\
\text { Theater }\end{array}$ & $\begin{array}{l}\text { Theater in der Königgrätzer- } \\
\text { straße, Theater in der Strese- } \\
\text { mannstraße }\end{array}$ & $\begin{array}{l}\text { Königgrätzer- } \\
\text { straße (seit } \\
1929 \text { Strese- } \\
\text { mannstraße) }\end{array}$ & 1908 & & 750 \\
\hline $\begin{array}{l}\text { Komödien- } \\
\text { haus }\end{array}$ & Neues Operetten-Theater & $\begin{array}{l}\text { Schiffbauer- } \\
\text { damm } 25\end{array}$ & 1908 & 1943 & 850 \\
\hline Admiralspalast & & $\begin{array}{l}\text { Friedrich- } \\
\text { straße }\end{array}$ & 1911 & & \\
\hline $\begin{array}{l}\text { Deutsches } \\
\text { Künstlerthea- } \\
\text { ter }\end{array}$ & & $\begin{array}{l}\text { Nürnberger } \\
\text { Straße 70/72 }\end{array}$ & 1911 & & 1050 \\
\hline $\begin{array}{l}\text { Deutsches } \\
\text { Opernhaus }\end{array}$ & Städtische Oper & $\begin{array}{l}\text { Bismarck- } \\
\text { straße 34-37 }\end{array}$ & 1912 & $\begin{array}{l}1927 \text { Kino, } \\
1933 \text { ge- } \\
\text { schlossen }\end{array}$ & 2300 \\
\hline Volksbühne & Theater am Bülowplatz & Bülowplatz & 1914 & & 2000 \\
\hline $\begin{array}{l}\text { Intimes } \\
\text { Theater }\end{array}$ & & Bülowstraße & 1916 & & \\
\hline Die Tribüne & & $\begin{array}{l}\text { Berliner } \\
\text { Straße } 37\end{array}$ & 1919 & & 295 \\
\hline Eden-Theater & & & 1919 & & 1000 \\
\hline $\begin{array}{l}\text { Großes Schau- } \\
\text { spielhaus }\end{array}$ & $\begin{array}{l}\text { zuvor als Zirkus Renz, } \\
\text { Zirkus Schumann }\end{array}$ & Am Zirkus & 1919 & & 3200 \\
\hline $\begin{array}{l}\text { Neues Theater } \\
\text { am Zoo }\end{array}$ & $\begin{array}{l}\text { Deutsches Volkstheater } \\
(1929-30)\end{array}$ & $\begin{array}{l}\text { Jebens- } \\
\text { straße } 2\end{array}$ & 1920 & 1937 & \\
\hline $\begin{array}{l}\text { Schloßpark- } \\
\text { Theater }\end{array}$ & & $\begin{array}{l}\text { Schloß- } \\
\text { straße } 48\end{array}$ & 1921 & 1935 Kino & 500 \\
\hline
\end{tabular}




\begin{tabular}{lllc}
\hline Name & Alternative Name & Ort & Eröffnet Geschlossen Sitzplätze \\
\hline $\begin{array}{l}\text { Renaissance- } \\
\text { Theater }\end{array}$ & $\begin{array}{l}\text { Hardenberg- } \\
\text { straße 6 }\end{array}$ & 1922 & 620 \\
$\begin{array}{l}\text { Theater am } \\
\begin{array}{l}\text { Kurfürsten- } \\
\text { damm }\end{array}\end{array}$ & $\begin{array}{l}\text { Kurfürsten- } \\
\text { damm }\end{array}$ & 1922 & 798 \\
$\begin{array}{l}\text { Komödie am } \\
\begin{array}{l}\text { Kurfürsten- } \\
\text { damm }\end{array}\end{array}$ & $\begin{array}{l}\text { Kurfürsten- } \\
\text { damm }\end{array}$ & 1924 & 450 \\
$\begin{array}{l}\text { Theater in der } \\
\text { Klosterstraße }\end{array}$ & $\begin{array}{l}\text { Kloster- } \\
\text { straße 43 }\end{array}$ & 1924 & 800 \\
\hline
\end{tabular}

Quelle: Neuer Theater-Almanach; Deutsches Bühnenjahrbuch; Wediggen, Geschichte der Berliner Theater; WAHNRAU, Berlin; LeONHARDT, Piktoral-Dramaturgie, S. 315-344; ITODA, Berlin \& Tokio, S. 148-151. 


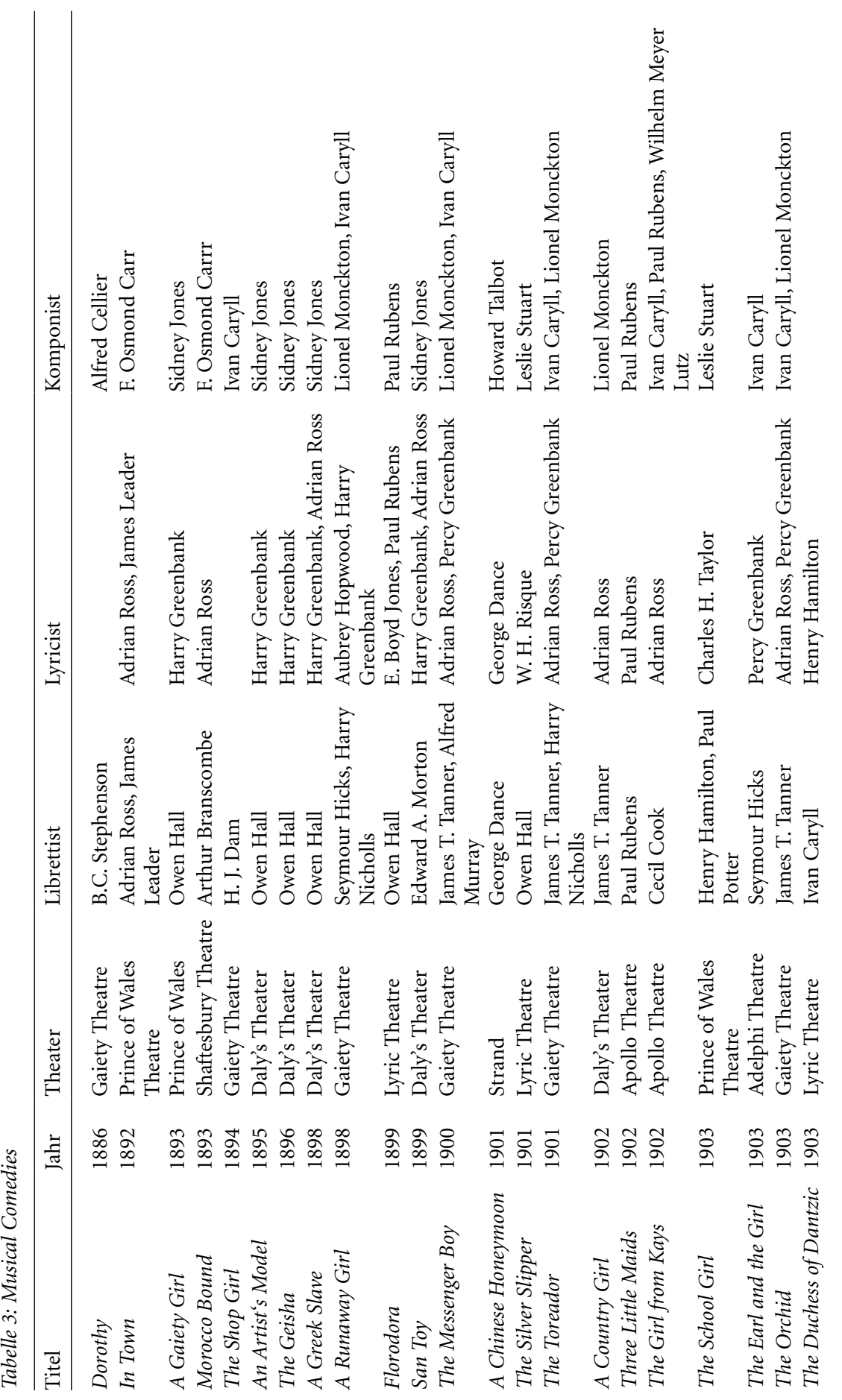




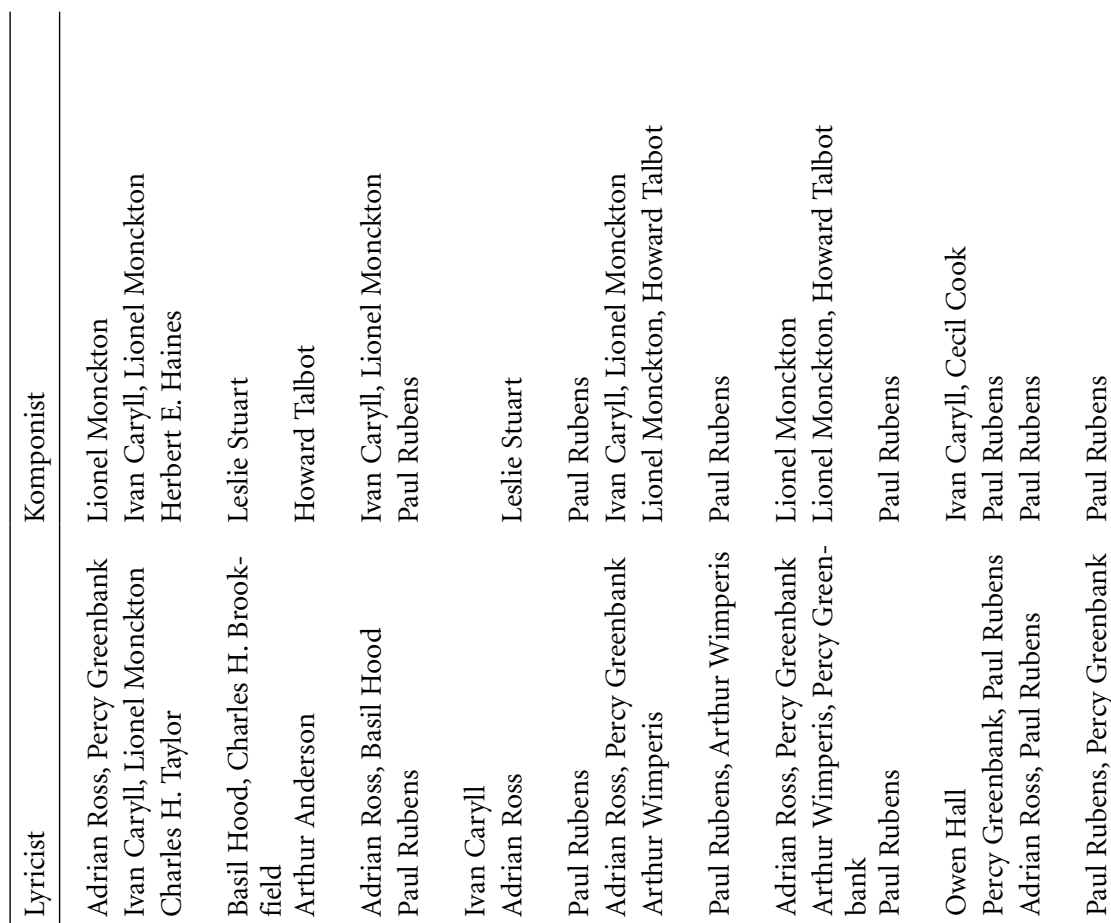

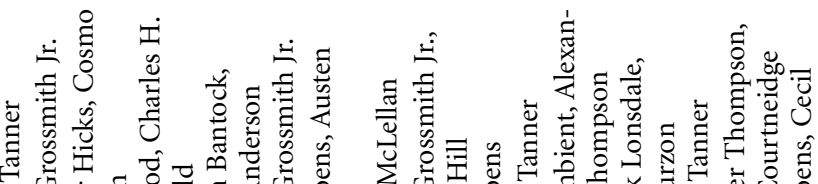

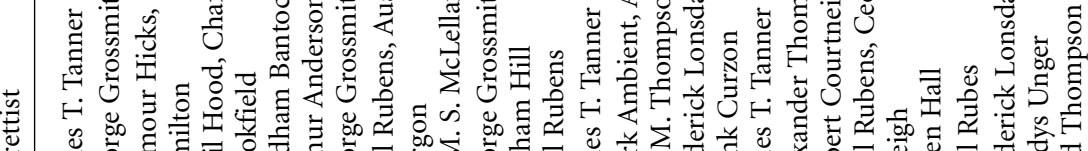

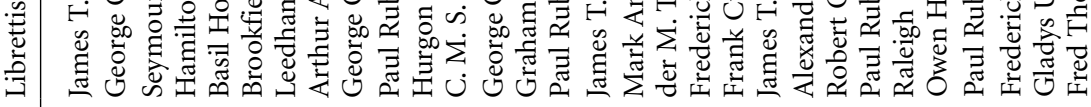

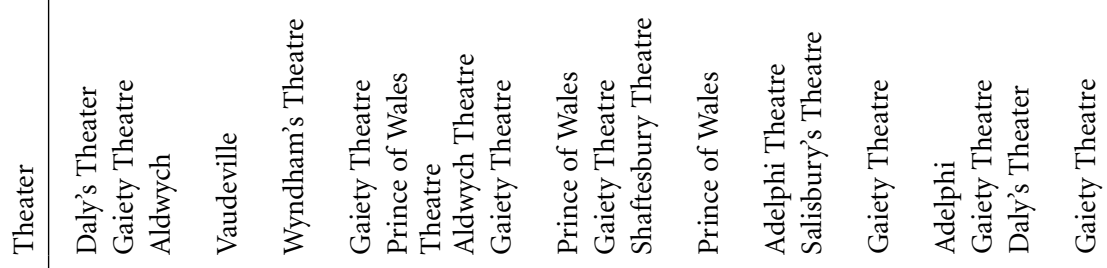

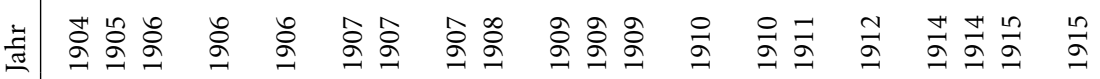

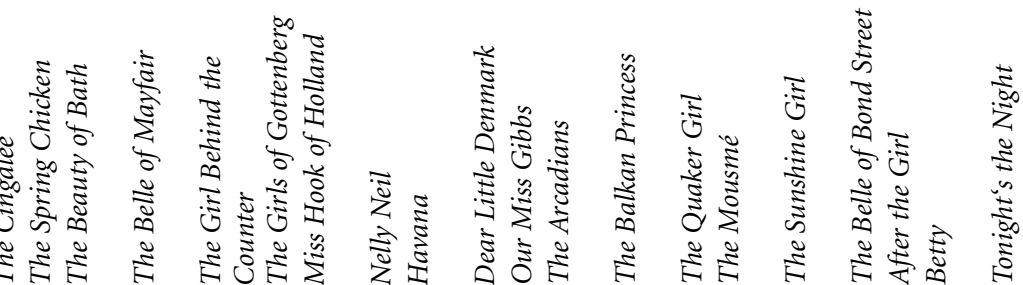




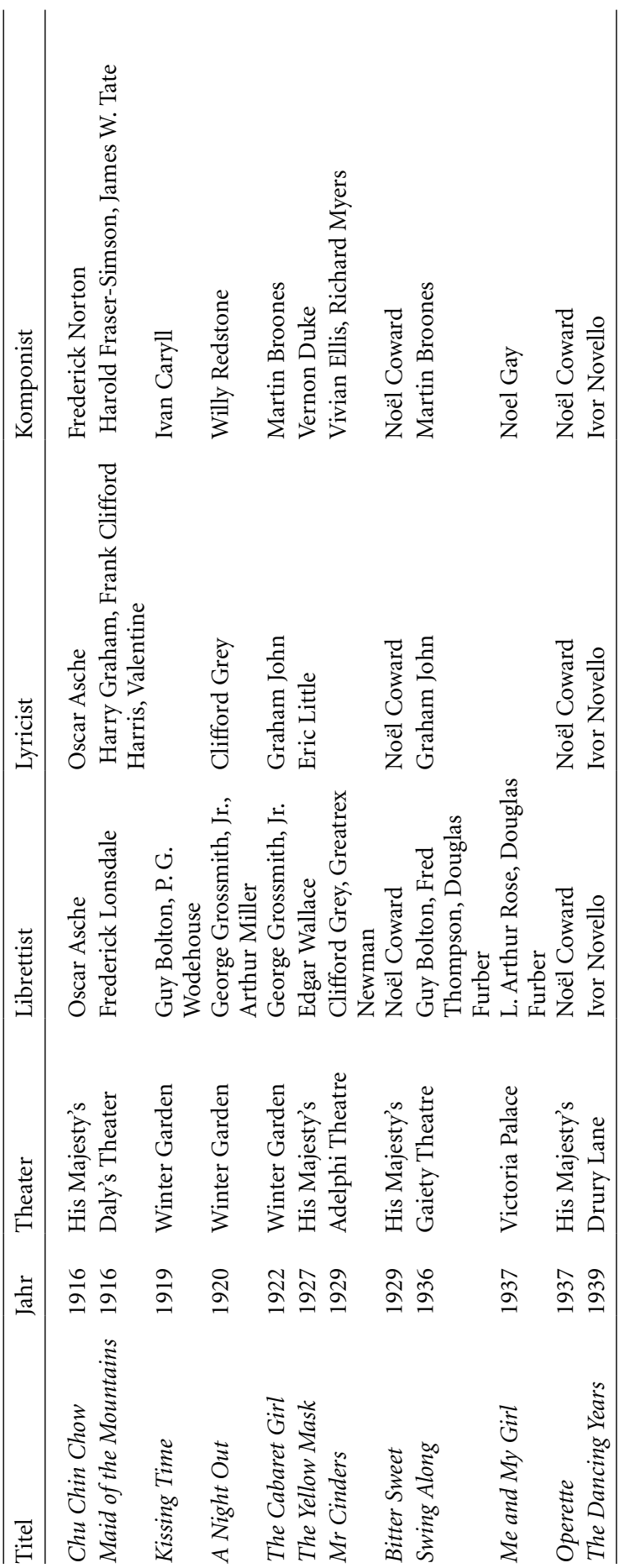


Tabelle 4: Revuen (London)

\begin{tabular}{|c|c|c|c|c|}
\hline Titel & Jahr & Theater & Librettist & Komponist \\
\hline $\begin{array}{l}\text { Everybody's } \\
\text { doing it }\end{array}$ & 1912 & Empire Theatre & $\begin{array}{l}\text { George Grossmith, C.H. } \\
\text { Bovill }\end{array}$ & Irving Berlin \\
\hline Hullo, Ragtime! & 1912 & $\begin{array}{l}\text { London } \\
\text { Hippodrome }\end{array}$ & $\begin{array}{l}\text { Max Pemberton, Albert } \\
\text { de Courville }\end{array}$ & Louis Hirsch u. a. \\
\hline Hullo, Tango! & 1913 & $\begin{array}{l}\text { London } \\
\text { Hippodrome }\end{array}$ & $\begin{array}{l}\text { Max Pemberton, Albert } \\
\text { de Courville }\end{array}$ & Louis Hirsch u. a. \\
\hline The Passing Show & 1914 & Palace Theatre & Arthur Wimperis & Herman Finck \\
\hline Jig-Saw! & 1914 & $\begin{array}{l}\text { London } \\
\text { Hippodrome }\end{array}$ & $\begin{array}{l}\text { Albert de Courville, Wal } \\
\text { Pink, Edgar Wallace }\end{array}$ & $\begin{array}{l}\text { Edward Chappelle, } \\
\text { Edward A. Horan }\end{array}$ \\
\hline Odds and Ends & 1914 & $\begin{array}{l}\text { Ambassadors } \\
\text { Theatre }\end{array}$ & Harry Grattan & \\
\hline Joy-Land! & 1914 & $\begin{array}{l}\text { London } \\
\text { Hippodrome }\end{array}$ & $\begin{array}{l}\text { Albert de Courville, Wal } \\
\text { Pink }\end{array}$ & Herman Darewski \\
\hline Watch your step & 1915 & Empire Theatre & $\begin{array}{l}\text { Harry B. Smith, Harry } \\
\text { Grattan }\end{array}$ & Irving Berlin \\
\hline Push and Go & 1915 & $\begin{array}{l}\text { London } \\
\text { Hippodrome }\end{array}$ & $\begin{array}{l}\text { Albert de Courville, F.W. } \\
\text { Marks }\end{array}$ & Herman Darewski \\
\hline Shell Out & 1915 & Comedy Theatre & $\begin{array}{l}\text { Albert de Courville, } \\
\text { Wal Pink }\end{array}$ & Herman Darewski \\
\hline Bric-a-Brac & 1915 & Palace Theatre & $\begin{array}{l}\text { Arthur Wimperis, } \\
\text { Basil Hood }\end{array}$ & $\begin{array}{l}\text { Lionel Monckton, } \\
\text { Herman Finck }\end{array}$ \\
\hline $\begin{array}{l}\text { The Bing Boys are } \\
\text { Here }\end{array}$ & 1916 & $\begin{array}{l}\text { Alhambra } \\
\text { Theatre }\end{array}$ & $\begin{array}{l}\text { George Grossmith, } \\
\text { Fred Thompson }\end{array}$ & Nat D. Ayer \\
\hline Pell-Mell & 1916 & $\begin{array}{l}\text { Ambassadors } \\
\text { Theatre }\end{array}$ & $\begin{array}{l}\text { Fred Thompson, Morris } \\
\text { Harvey }\end{array}$ & Nat D. Ayer \\
\hline Razzle-Dazzle & 1916 & Drury Lane & $\begin{array}{l}\text { A. de Courville, W. Pink, } \\
\text { B. MacDonald Hastings }\end{array}$ & $\begin{array}{l}\text { Herman Darewski, } \\
\text { Manuel Klein }\end{array}$ \\
\hline Zig-Zag! & 1917 & $\begin{array}{l}\text { London } \\
\text { Hippodrome }\end{array}$ & $\begin{array}{l}\text { Albert de Courville, Wal } \\
\text { Pink, George Arnold }\end{array}$ & Dave Stamper \\
\hline A to $\mathrm{Z}$ & 1917 & $\begin{array}{l}\text { Prince of Wales } \\
\text { Theatre }\end{array}$ & $\begin{array}{l}\text { Dion Titheradge, Ronald } \\
\text { Jeans, Helen Trix }\end{array}$ & $\begin{array}{l}\text { Ivor Novello, Helen } \\
\text { Trix }\end{array}$ \\
\hline Box O`Tricks! & 1918 & $\begin{array}{l}\text { London } \\
\text { Hippodrome }\end{array}$ & $\begin{array}{l}\text { Albert de Courville, } \\
\text { Wal Pink }\end{array}$ & $\begin{array}{l}\text { Dave Stamper, } \\
\text { Frederick Chappelle. }\end{array}$ \\
\hline Hullo, America! & 1918 & Palace Theatre & John Hastings Turner & Herman Finck \\
\hline U.S. & 1918 & $\begin{array}{l}\text { Ambassadors } \\
\text { Theatre }\end{array}$ & Harry Grattan & $\begin{array}{l}\text { Grant Clarke, Milton } \\
\text { Ager, George W. Meyer }\end{array}$ \\
\hline$B u z z-B u z z$ & 1918 & $\begin{array}{l}\text { Vaudeville } \\
\text { Theatre }\end{array}$ & $\begin{array}{l}\text { Arthur Wimperis, } \\
\text { Ronald Jeans }\end{array}$ & Herman Darewski \\
\hline The Whirligig & 1919 & Palace Theatre & $\begin{array}{l}\text { Albert de Courville, } \\
\text { Wal Pink, Edgar Wallace }\end{array}$ & Frederick Chappelle \\
\hline League of Notions & 1921 & $\begin{array}{l}\text { New Oxford } \\
\text { Theatre }\end{array}$ & $\begin{array}{l}\text { John Murray Anderson, } \\
\text { Augustus Barratt }\end{array}$ & $\begin{array}{l}\text { Milton Ager, Augustus } \\
\text { Barratt, Helen Trix }\end{array}$ \\
\hline London Calling! & 1923 & $\begin{array}{l}\text { Duke of York's } \\
\text { Theatre }\end{array}$ & Noël Coward & Noël Coward \\
\hline $\begin{array}{l}\text { Charlot's Revue of } \\
1924\end{array}$ & 1924 & $\begin{array}{l}\text { Prince of Wales } \\
\text { Theatre }\end{array}$ & Ronald Jeans & Verschiedene \\
\hline $\begin{array}{l}\text { Charlot's Revue } \\
1925\end{array}$ & 1925 & $\begin{array}{l}\text { Prince of Wales } \\
\text { Theatre }\end{array}$ & Ronald Jeans & Verschiedene \\
\hline Cochran's Revue & 1926 & London Pavilion & Ronald Jeans & Verschiedene \\
\hline This Year of Grace & 1928 & London Pavilion & Noël Coward & Noël Coward \\
\hline $\begin{array}{l}\text { Cochran's } 1931 \\
\text { Revue }\end{array}$ & 1931 & London Pavilion & Noël Coward & Noël Coward \\
\hline
\end{tabular}


Tabelle 5: Berliner Operetten

\begin{tabular}{|c|c|c|c|c|}
\hline Titel & Jahr & Theater & Librettist & Komponist \\
\hline Eine tolle Nacht & 1895 & Central-Theater & $\begin{array}{l}\text { Julius Freund, Wilhelm } \\
\text { Mannstaedt }\end{array}$ & Julius Einödshofer \\
\hline Venus auf Erden & 1897 & Apollo-Theater & Heinrich Bolten-Bäckers & Paul Lincke \\
\hline Die verkehrte Welt & 1899 & Metropol-Theater & Julius Freund & $\begin{array}{l}\text { Gaston Serpelle, } \\
\text { Julius Einödshofer }\end{array}$ \\
\hline Frau Luna & 1899 & Apollo-Theater & Heinrich Bolten-Bäckers & Paul Lincke \\
\hline Im Reich des Indra & 1899 & Apollo-Theater & Heinrich Bolten-Bäckers & Paul Lincke \\
\hline $\begin{array}{l}\text { Der Zauberer vom } \\
\text { Nil }\end{array}$ & 1900 & Metropol-Theater & Julius Freund & \\
\hline Fräulein Loreley & 1900 & Apollo-Theater & Heinrich Bolten-Bäckers & Paul Lincke \\
\hline Madame Sherry & 1902 & Central-Theater & $\begin{array}{l}\text { Maurice Ordonneau, } \\
\text { Benno Jacobson }\end{array}$ & Hugo Felix \\
\hline Lysistrata & 1902 & Apollo-Theater & Heinrich Bolten-Bäckers & Paul Lincke \\
\hline Berliner Luft & 1904 & Apollo-Theater & Heinrich Bolten-Bäckers & Paul Lincke \\
\hline Prinzeß Rosine & 1905 & Apollo-Theater & Heinrich Bolten-Bäckers & Paul Lincke \\
\hline $\begin{array}{l}\text { Das bummelnde } \\
\text { Berlin }\end{array}$ & 1906 & Apollo-Theater & Benno Jacobson & Rudolf Nelson \\
\hline $\begin{array}{l}\text { Die oberen } \\
\text { Zehntausend }\end{array}$ & 1909 & Metropol-Theater & Julius Freund & Gustave Kerker \\
\hline Polnische Wirtschaft & 1910 & Thalia-Theater & $\begin{array}{l}\text { Jean Kren, Alfred } \\
\text { Schönfeld }\end{array}$ & Jean Gilbert \\
\hline Die keusche Susanne & 1911 & $\begin{array}{l}\text { Neues Operetten- } \\
\text { theater }\end{array}$ & Georg Okonowski & Jean Gilbert \\
\hline Die moderne Eva & 1911 & $\begin{array}{l}\text { Neues Operetten- } \\
\text { theater }\end{array}$ & Georg Okonowski & $\begin{array}{l}\text { Jean Gilbert, Alfred } \\
\text { Schönfeld }\end{array}$ \\
\hline $\begin{array}{l}\text { Hoheit amüsiert } \\
\text { sich }\end{array}$ & 1911 & Metropol-Theater & Julius Freund & Rudolf Nelson \\
\hline Der liebe Augustin & 1912 & $\begin{array}{l}\text { Neues Theater am } \\
\text { Zoo }\end{array}$ & $\begin{array}{l}\text { Rudolf Bernaue, } \\
\text { Ernst Welisch }\end{array}$ & Leo Fall \\
\hline Autoliebchen & 1912 & Thaliatheater & $\begin{array}{l}\text { Jean Kren, Alfred } \\
\text { Schönfeld }\end{array}$ & Jean Gilbert \\
\hline $\begin{array}{l}\text { Ein aufgelegtes } \\
\text { Geschäft }\end{array}$ & 1912 & Komische Oper & $\begin{array}{l}\text { Herman Frey, F. W. } \\
\text { Hardt }\end{array}$ & Walter Kollo \\
\hline Filmzauber & 1912 & Berliner Theater & $\begin{array}{l}\text { R. Bernauer, } \\
\text { R. Schanzer }\end{array}$ & $\begin{array}{l}\text { Walter Kollo, Willy } \\
\text { Bredschneider }\end{array}$ \\
\hline Puppchen & 1912 & Thaliatheater & $\begin{array}{l}\text { Jean Kren, Kurt Kraatz, } \\
\text { Alfred Schönfeld }\end{array}$ & Jean Gilbert \\
\hline Die Kino-Königin & 1913 & Metropol-Theater & $\begin{array}{l}\text { Georg Okonowski, } \\
\text { Julius Freund }\end{array}$ & Jean Gilbert \\
\hline $\begin{array}{l}\text { Die Reise um die } \\
\text { Erde in } 40 \text { Tagen }\end{array}$ & 1913 & Metropol-Theater & Julius Freund & Jean Gilbert \\
\hline Die Tangoprinzessin & 1913 & Thalia-Theater & $\begin{array}{l}\text { Jean Kren, Alfred } \\
\text { Schönfeld }\end{array}$ & Jean Gilbert \\
\hline Wie einst im Mai & 1913 & Berliner Theater & $\begin{array}{l}\text { Rudolf Bernauer, } \\
\text { Rudolph Schanzer }\end{array}$ & $\begin{array}{l}\text { Walter Kollo, Willy } \\
\text { Bredschneider }\end{array}$ \\
\hline Fräulein Tra-la-la & 1913 & $\begin{array}{l}\text { Neues Luisen- } \\
\text { Theater }\end{array}$ & $\begin{array}{l}\text { Georg Okonkowski, } \\
\text { Leo Leipziger }\end{array}$ & Jean Gilbert \\
\hline Jung-England & 1914 & $\begin{array}{l}\text { Montis Operetten- } \\
\text { theater }\end{array}$ & $\begin{array}{l}\text { Rudolf Bernauer, } \\
\text { Ernst Welisch }\end{array}$ & Leo Fall \\
\hline
\end{tabular}




\begin{tabular}{|c|c|c|c|c|}
\hline Titel & Jahr & Theater & Librettist & Komponist \\
\hline Extrablätter & 1914 & Berliner Theater & $\begin{array}{l}\text { Bredschneider, Rudolph } \\
\text { Schanzer }\end{array}$ & Walter Kollo \\
\hline Immer feste druff & 1914 & $\begin{array}{l}\text { Theater am } \\
\text { Nollendorfplatz }\end{array}$ & $\begin{array}{l}\text { Hermann Haller, Willi } \\
\text { Wolff }\end{array}$ & Walter Kollo \\
\hline $\begin{array}{l}\text { Wenn der Frühling } \\
\text { kommt }\end{array}$ & 1914 & Thaliatheater & $\begin{array}{l}\text { Georg Okonowski, Jean } \\
\text { Kren }\end{array}$ & $\begin{array}{l}\text { Jean Gilbert, Alfred } \\
\text { Schönfeld }\end{array}$ \\
\hline $\begin{array}{l}\text { Die Frau im } \\
\text { Hermelin }\end{array}$ & 1919 & $\begin{array}{l}\text { Theater des } \\
\text { Westens }\end{array}$ & $\begin{array}{l}\text { Rudolf Schanzer, Ernst } \\
\text { Welisch }\end{array}$ & Jean Gilbert \\
\hline Der letzte Walzer & 1920 & Berliner Theater & $\begin{array}{l}\text { Julius Brammer, Alfred } \\
\text { Grünwald }\end{array}$ & Oscar Straus \\
\hline Wenn Liebe erwacht & 1920 & $\begin{array}{l}\text { Theater am } \\
\text { Nollendorfplatz }\end{array}$ & $\begin{array}{l}\text { Herman Haller, Ridea- } \\
\text { mus }\end{array}$ & Eduard Künneke \\
\hline $\begin{array}{l}\text { Der Vetter aus } \\
\text { Dingsda }\end{array}$ & 1921 & $\begin{array}{l}\text { Theater am } \\
\text { Nollendorfplatz }\end{array}$ & $\begin{array}{l}\text { Hermann Haller, Ridea- } \\
\text { mus (=Fritz Oliven) }\end{array}$ & Eduard Künneke \\
\hline $\begin{array}{l}\text { Madame Pompa- } \\
\text { dour }\end{array}$ & 1922 & Berliner Theater & $\begin{array}{l}\text { Rudolf Schanzer, Ernst } \\
\text { Welisch }\end{array}$ & Leo Fall \\
\hline $\begin{array}{l}\text { Der Fürst von Pap- } \\
\text { penheim }\end{array}$ & 1923 & $\begin{array}{l}\text { Deutsches } \\
\text { Künstlertheater }\end{array}$ & $\begin{array}{l}\text { Franz Arnold, Ernst } \\
\text { Bach }\end{array}$ & Hugo Hirsch \\
\hline Wie einst im Mai & 1927 & $\begin{array}{l}\text { Großes Schauspiel- } \\
\text { haus }\end{array}$ & $\begin{array}{l}\text { Rudolf Bernauer, Ernst } \\
\text { Welisch }\end{array}$ & $\begin{array}{l}\text { Walter Kollo, Willy } \\
\text { Bredschneider }\end{array}$ \\
\hline Der Mikado & 1927 & $\begin{array}{l}\text { Großes Schauspiel- } \\
\text { haus }\end{array}$ & & \\
\hline Casanova & 1928 & $\begin{array}{l}\text { Großes Schauspiel- } \\
\text { haus }\end{array}$ & $\begin{array}{l}\text { Rudolph Schanzer, Ernst } \\
\text { Welisch }\end{array}$ & Johann Strauß \\
\hline Friederike & 1928 & Metropol-Theater & $\begin{array}{l}\text { Ludwig Herzer, Fritz } \\
\text { Löhner-Beda }\end{array}$ & Franz Lehár \\
\hline $\begin{array}{l}\text { Die Dreigroschen- } \\
\text { oper }\end{array}$ & 1928 & $\begin{array}{l}\text { Theater am Schiff- } \\
\text { bauerdamm }\end{array}$ & Bertolt Brecht & Kurt Weill \\
\hline $\begin{array}{l}\text { Das Land des } \\
\text { Lächelns }\end{array}$ & 1929 & Metropol-Theater & $\begin{array}{l}\text { Ludwig Herzer, Fritz } \\
\text { Löhner-Beda }\end{array}$ & Franz Lehár \\
\hline Die drei Musketiere & 1929 & $\begin{array}{l}\text { Großes Schauspiel- } \\
\text { haus }\end{array}$ & $\begin{array}{l}\text { Rudolph Schanzer, Ernst } \\
\text { Benatzky }\end{array}$ & Welisch, Ralph \\
\hline Schön ist die Welt & 1930 & Metropol-Theater & $\begin{array}{l}\text { Ludwig Herzer, Fritz } \\
\text { Löhner-Beda }\end{array}$ & Franz Lehár \\
\hline Die lustige Witwe & 1930 & $\begin{array}{l}\text { Großes Schauspiel- } \\
\text { haus }\end{array}$ & Victor Léon, Leo Stein & Franz Lehár \\
\hline Im Weißen Rößl & 1930 & $\begin{array}{l}\text { Großes Schauspiel- } \\
\text { haus }\end{array}$ & $\begin{array}{l}\text { Hans Müller, Robert } \\
\text { Gilbert }\end{array}$ & $\begin{array}{l}\text { Ralph Benatzky, } \\
\text { Robert Stolz, Bruno } \\
\text { Granichstaedten, } \\
\text { Robert Gilbert }\end{array}$ \\
\hline Die Dubarry & 1931 & Admiralspalast & $\begin{array}{l}\text { Paul Knepler, Ignaz } \\
\text { Michael Welleminsky, } \\
\text { Hans Martin Cremer }\end{array}$ & $\begin{array}{l}\text { Carl Millöcker, Theo } \\
\text { Mackeben }\end{array}$ \\
\hline $\begin{array}{l}\text { Die Blume von } \\
\text { Hawai }\end{array}$ & 1931 & Metropol-Theater & $\begin{array}{l}\text { Alfred Grünwald, Fritz } \\
\text { Löhner-Beda }\end{array}$ & Paul Abraham \\
\hline $\begin{array}{l}\text { Eine Frau, die weiß, } \\
\text { was sie will }\end{array}$ & 1932 & Metropol-Theater & Alfred Grünwald & Oscar Straus \\
\hline Der Ball im Savoy & 1932 & Metropol-Theater & $\begin{array}{l}\text { Alfred Grünwald, Fritz } \\
\text { Löhner-Beda }\end{array}$ & Paul Abraham \\
\hline
\end{tabular}


Tabelle 6: Berliner Revuen

\begin{tabular}{|c|c|c|c|c|}
\hline Titel & Jahr & Theater & Librettist & Komponist \\
\hline Im Paradies der Damen & 1898 & $\begin{array}{l}\text { Metropol- } \\
\text { Theater }\end{array}$ & Julius Freund & Julius Einödshofer \\
\hline Berlin lacht & 1899 & $\begin{array}{l}\text { Metropol- } \\
\text { Theater }\end{array}$ & Julius Freund & Julius Einödshofer \\
\hline Rund um Berlin & 1899 & $\begin{array}{l}\text { Metropol- } \\
\text { Theater }\end{array}$ & Julius Freund & Julius Einödshofer \\
\hline Berlin bleibt Berlin & 1902 & $\begin{array}{l}\text { Metropol- } \\
\text { Theater }\end{array}$ & Julius Freund & \\
\hline Neuestes! Allerneuestes! & 1903 & $\begin{array}{l}\text { Metropol- } \\
\text { Theater }\end{array}$ & Julius Freund & Victor Hollaender \\
\hline Ein tolles Jahr & 1904 & $\begin{array}{l}\text { Metropol- } \\
\text { Theater }\end{array}$ & Julius Freund & Victor Hollaender \\
\hline Auf in's Metropol! & 1905 & $\begin{array}{l}\text { Metropol- } \\
\text { Theater }\end{array}$ & Julius Freund & Victor Hollaender \\
\hline Der Teufel lacht dazu & 1906 & $\begin{array}{l}\text { Metropol- } \\
\text { Theater }\end{array}$ & Julius Freund & Victor Hollaender \\
\hline Das muß man seh'n! & 1907 & $\begin{array}{l}\text { Metropol- } \\
\text { Theater }\end{array}$ & Julius Freund & Victor Hollaender \\
\hline Donnerwetter - tadellos! & 1908 & $\begin{array}{l}\text { Metropol- } \\
\text { Theater }\end{array}$ & Julius Freund & Paul Lincke \\
\hline Hallo! Die große Revue & 1909 & $\begin{array}{l}\text { Metropol- } \\
\text { Theater }\end{array}$ & Julius Freund & Paul Lincke \\
\hline Hurra! wir leben noch! & 1910 & $\begin{array}{l}\text { Metropol- } \\
\text { Theater }\end{array}$ & Julius Freund & Victor Hollaender \\
\hline Die Nacht von Berlin & 1911 & $\begin{array}{l}\text { Metropol- } \\
\text { Theater }\end{array}$ & Julius Freund & Victor Hollaender \\
\hline $\begin{array}{l}\text { Chauffeur - in's } \\
\text { Metropol! }\end{array}$ & 1912 & $\begin{array}{l}\text { Metropol- } \\
\text { Theater }\end{array}$ & Julius Freund & Rudolf Nelson \\
\hline Woran wir denken & 1914 & $\begin{array}{l}\text { Metropol- } \\
\text { Theater }\end{array}$ & $\begin{array}{l}\text { Franz Arnold, Walter } \\
\text { Turszinsky }\end{array}$ & Jean Gilbert \\
\hline Die Welt geht unter & 1918 & Komische Oper & & \\
\hline Die Welt im Jahr 200 & 1919 & Komische Oper & & \\
\hline Rund um die Welt & 1920 & Komische Oper & & \\
\hline Der Herr der Welt & 1921 & Komische Oper & & \\
\hline Europa spricht davon & 1922 & Komische Oper & & \\
\hline Drunter und Drüber & 1923 & Admiralspalast & $\begin{array}{l}\text { Hermann Haller, Ri- } \\
\text { deamus, Willi Wolff }\end{array}$ & Walter Kollo \\
\hline Die Welt ohne Schleier & 1923 & Komische Oper & & \\
\hline An Alle & 1924 & $\begin{array}{l}\text { Großes } \\
\text { Schauspielhaus }\end{array}$ & $\begin{array}{l}\text { Fritz Beda, Willy Pra- } \\
\text { ger }\end{array}$ & $\begin{array}{l}\text { Ralph Benatzky, } \\
\text { Rudolf Nelson, } \\
\text { Irving Berlin }\end{array}$ \\
\hline Noch und Noch & 1924 & Admiralspalast & $\begin{array}{l}\text { Hermann Haller, Ri- } \\
\text { deamus, Willi Wolff }\end{array}$ & Walter Kollo \\
\hline $\begin{array}{l}\text { Das hat die Welt noch } \\
\text { nicht gesehn }\end{array}$ & 1924 & Komische Oper & & \\
\hline Für Dich & 1925 & $\begin{array}{l}\text { Großes } \\
\text { Schauspielhaus }\end{array}$ & Ralph Benatzky & Ralph Benatzky \\
\hline
\end{tabular}




\begin{tabular}{|c|c|c|c|c|}
\hline Titel & Jahr & Theater & Librettist & Komponist \\
\hline Achtung, Welle 505 & 1925 & Admiralspalast & $\begin{array}{l}\text { Hermann Haller, Ri- } \\
\text { deamus, Willi Wolff }\end{array}$ & Walter Kollo \\
\hline Von $A$ bis $Z$ & 1925 & Komische Oper & & \\
\hline Das lachende Berlin & 1925 & Komische Oper & & \\
\hline Berlin ohne Hemd & 1926 & Komische Oper & & \\
\hline An und Aus & 1926 & Admiralspalast & & \\
\hline Wieder Metropol & 1926 & $\begin{array}{l}\text { Metropol- } \\
\text { Theater }\end{array}$ & $\begin{array}{l}\text { Artur Rebner, Willi } \\
\text { Hagen }\end{array}$ & Hugo Hirsch \\
\hline Von Mund zu Mund & 1926 & $\begin{array}{l}\text { Großes } \\
\text { Schauspielhaus }\end{array}$ & & \\
\hline Wann und Wo & 1927 & Admiralspalast & $\begin{array}{l}\text { Hermann Haller, Ri- } \\
\text { deamus, Willi Wolff }\end{array}$ & Walter Kollo \\
\hline Die Welt applaudiert & 1927 & Komische Oper & & \\
\hline Alles nackt & 1927 & Komische Oper & & \\
\hline Zieh dich aus & 1928 & Komische Oper & & \\
\hline Dreimädlerhaus & 1928 & $\begin{array}{l}\text { Großes } \\
\text { Schauspielhaus }\end{array}$ & & \\
\hline Schön und Schick & 1928 & Admiralspalast & & \\
\hline $\begin{array}{l}\text { Donnerwetter }-1000 \\
\text { Frauen }\end{array}$ & 1928 & Komische Oper & & \\
\hline Tausend nackte Frauen & 1928 & Komische Oper & & \\
\hline Häuser der Liebe & 1928 & Komische Oper & & \\
\hline Bitte einsteigen & 1928 & $\begin{array}{l}\text { Theater des } \\
\text { Westens }\end{array}$ & $\begin{array}{l}\text { Günther Bibo, } \\
\text { Charlie Roellinghoff, } \\
\text { Friedrich Hollaender }\end{array}$ & Friedrich Hollaender \\
\hline Es liegt in der Luft & 1928 & & $\begin{array}{l}\text { Marcellus Schiffer, } \\
\text { Mischa Spoliansky }\end{array}$ & \\
\hline $\begin{array}{l}\text { Paradies der süßen } \\
\text { Frauen }\end{array}$ & 1929 & Komische Oper & & \\
\hline Der liebe Augustin & 1929 & $\begin{array}{l}\text { Großes } \\
\text { Schauspielhaus }\end{array}$ & & \\
\hline Von Bettchen zu Bettchen & 1929 & Komische Oper & & \\
\hline
\end{tabular}


Tabelle 7: Transfer London - Berlin

\begin{tabular}{|c|c|c|c|c|c|}
\hline Originaltitel & Theater & Jahr & Deutscher Titel & Theater & Jahr \\
\hline A Gaiety Girl & $\begin{array}{l}\text { Prince of Wales } \\
\text { Theatre }\end{array}$ & 14.10 .1893 & Ein fideles Corps & $\begin{array}{l}\text { Adolf-Ernst- } \\
\text { Theater }\end{array}$ & 1894 \\
\hline Morrocco Bound & $\begin{array}{l}\text { Shaftesbury } \\
\text { Theatre }\end{array}$ & 13.4 .1893 & Unbekannt & $\begin{array}{l}\text { Theater Unter } \\
\text { den Linden }\end{array}$ & 16.2 .1895 \\
\hline The Geisha & Daly's Theatre & 25.4 .1896 & Die Geisha & $\begin{array}{l}\text { Lessing } \\
\text { Theater }\end{array}$ & 1.5 .1897 \\
\hline A Greek Slave & Daly's Theatre & 8.6.1898 & $\begin{array}{l}\text { Der griechische } \\
\text { Sklave }\end{array}$ & $\begin{array}{l}\text { Central- } \\
\text { Theater }\end{array}$ & 19.2.1899 \\
\hline $\begin{array}{l}\text { A Runaway } \\
\text { Girl }\end{array}$ & Gaiety Theatre & 21.5 .1898 & Daisy & $\begin{array}{l}\text { Lessing- } \\
\text { Theater }\end{array}$ & 19.5 .1900 \\
\hline San Toy & Daly's Theatre & 21.10 .1899 & San Toy & $\begin{array}{l}\text { Central- } \\
\text { Theater }\end{array}$ & 2.2 .1901 \\
\hline $\begin{array}{l}\text { A Chinese } \\
\text { Honeymoon }\end{array}$ & $\begin{array}{l}\text { Royal Strand } \\
\text { Theatre }\end{array}$ & 16.10.1899 & $\begin{array}{l}\text { Chinesische } \\
\text { Flitterwochen }\end{array}$ & $\begin{array}{l}\text { Central- } \\
\text { Theater }\end{array}$ & 25.4.1903 \\
\hline $\begin{array}{l}\text { The Silver } \\
\text { Slipper }\end{array}$ & Lyric Theatre & 1.6 .1901 & $\begin{array}{l}\text { Der silberne } \\
\text { Pantoffel }\end{array}$ & $\begin{array}{l}\text { Central- } \\
\text { Theater? }\end{array}$ & 1902 \\
\hline Havana & Gaiety Theatre & 25.4 .1908 & Havanna & $\begin{array}{l}\text { Belle-Alliance- } \\
\text { Theater }\end{array}$ & 17. 10.1908 \\
\hline The Arcadians & $\begin{array}{l}\text { Shaftesbury } \\
\text { Theatre }\end{array}$ & 29.4.1909 & $\begin{array}{l}\text { Schwindelmeier } \\
\text { \& Co. }\end{array}$ & $\begin{array}{l}\text { Metropol- } \\
\text { Theater }\end{array}$ & 27.4 .1912 \\
\hline Mr Cinders & $\begin{array}{l}\text { Adelphi } \\
\text { Theatre }\end{array}$ & 11.2 .1929 & Jim und Jill & $\begin{array}{l}\text { Deutsches } \\
\text { Künstler- } \\
\text { theater }\end{array}$ & 16.9 .1930 \\
\hline
\end{tabular}


Tabelle 8: Transfer Berlin - London

\begin{tabular}{|c|c|c|c|c|c|}
\hline Originaltitel & Theater & Jahrg & Englischer Titel & Theater & Jahr \\
\hline $\begin{array}{l}\text { Eine tolle } \\
\text { Nacht }\end{array}$ & $\begin{array}{l}\text { Central- } \\
\text { Theater }\end{array}$ & 1.3 .1895 & The Circus Girl & $\begin{array}{l}\text { Gaiety } \\
\text { Theatre }\end{array}$ & 5.12 .1896 \\
\hline Frau Luna & Apollo-Theater & 23.3.1901 & $\begin{array}{l}\text { Castles in the } \\
\text { Air }\end{array}$ & Scala Theatre & 27.3.1911 \\
\hline $\begin{array}{l}\text { Madame } \\
\text { Sherry }\end{array}$ & $\begin{array}{l}\text { Central- } \\
\text { Theater }\end{array}$ & 1.11 .1902 & $\begin{array}{l}\text { Madame } \\
\text { Sherry }\end{array}$ & Apollo Theatre & 23.12 .1903 \\
\hline $\begin{array}{l}\text { Die keusche } \\
\text { Susanne }\end{array}$ & $\begin{array}{l}\text { Neues O } \\
\text { ten-Thea }\end{array}$ & 6.8 .1911 & $\begin{array}{l}\text { The Girl in the } \\
\text { Taxi }\end{array}$ & Lyric Theatre & 5.9.1912 \\
\hline $\begin{array}{l}\text { Der liebe } \\
\text { Augustin }\end{array}$ & $\begin{array}{l}\text { Neues Theater } \\
\text { am Zoo }\end{array}$ & 3.2 .1912 & $\begin{array}{l}\text { Princess } \\
\text { Caprice }\end{array}$ & $\begin{array}{l}\text { Shaftesbury } \\
\text { Theatre }\end{array}$ & 1.1 .1912 \\
\hline Autoliebchen & Thalia-Theater & 16.3 .1912 & Joy Ride Lady & New Theatre & 21.2 .1914 \\
\hline Filmzauber & $\begin{array}{l}\text { Berliner } \\
\text { Theater }\end{array}$ & 19.10 .1912 & $\begin{array}{l}\text { The Girl on the } \\
\text { Film }\end{array}$ & Gaiety Theatre & 5.4 .1913 \\
\hline $\begin{array}{l}\text { Die Kino- } \\
\text { Königin }\end{array}$ & $\begin{array}{l}\text { Metropol- } \\
\text { Theater }\end{array}$ & 8.3 .1913 & $\begin{array}{l}\text { The Cinema } \\
\text { Star }\end{array}$ & $\begin{array}{l}\text { Sha } \\
\text { The }\end{array}$ & 4.6.1914 \\
\hline $\begin{array}{l}\text { Fräulein } \\
\text { Tra-la-la }\end{array}$ & $\begin{array}{l}\text { Neues Luisen- } \\
\text { Theater }\end{array}$ & 15.11 .1913 & $\begin{array}{l}\text { Mam'selle Tra- } \\
\mathrm{La}-\mathrm{La}\end{array}$ & Lyric Theatre & 1.1 .1914 \\
\hline $\begin{array}{l}\text { Die Frau im } \\
\text { Hermelin }\end{array}$ & Thea & 23.8 .1919 & $\begin{array}{l}\text { The Lady of } \\
\text { the Rose }\end{array}$ & Daly's Theatre & 21.2 .1922 \\
\hline $\begin{array}{l}\text { Der letzte } \\
\text { Walzer }\end{array}$ & Berli & 12.2.1920 & The Last Waltz & Gaiety Theatre & 7.12.1922 \\
\hline $\begin{array}{l}\text { Wenn Liebe } \\
\text { erwacht }\end{array}$ & fplatz & 3.9.1920 & $\begin{array}{l}\text { Love's } \\
\text { Awakening }\end{array}$ & heatre & 19.4 .1921 \\
\hline $\begin{array}{l}\text { Der Vetter aus } \\
\text { Dingsda }\end{array}$ & $\begin{array}{l}\text { Theater am } \\
\text { Nollendorfplatz }\end{array}$ & 1 & $\begin{array}{l}\text { The Cousin } \\
\text { from Nowhere }\end{array}$ & $\begin{array}{l}\text { Prince's Theat- } \\
\text { re }\end{array}$ & 1923 \\
\hline $\begin{array}{l}\text { Madame } \\
\text { Pompadour }\end{array}$ & $\begin{array}{l}\text { Berliner } \\
\text { Theater }\end{array}$ & 9. & $\begin{array}{l}\text { Madame } \\
\text { Pompadour }\end{array}$ & Daly's Theatre & 923 \\
\hline $\begin{array}{l}\text { Der Fürst } \\
\text { Pappenhe }\end{array}$ & $\begin{array}{l}\text { Deuts } \\
\text { Küns }\end{array}$ & 16.2 .1923 & Toni & bury & 12.5 .1924 \\
\hline Mädi & $\begin{array}{l}\text { Berliner } \\
\text { Theater }\end{array}$ & 1.4 .1923 & The Blue Train & $\begin{array}{l}\text { Prince of } \\
\text { Wales's }\end{array}$ & 22.5 .1927 \\
\hline Casanova & $\begin{array}{l}\text { Großes Schau- } \\
\text { spielhaus }\end{array}$ & 1.9.1928 & Casanova & $\begin{array}{l}\text { Coliseum } \\
\text { Theatre }\end{array}$ & 24.5.1932 \\
\hline Friederike & $\begin{array}{l}\text { Metropol- } \\
\text { Theater }\end{array}$ & 28.10 .1928 & Frede & Palace Theatre & 9.9 .1930 \\
\hline $\begin{array}{l}\text { Das Land des } \\
\text { Lächelns }\end{array}$ & $\begin{array}{l}\text { Metropol- } \\
\text { Theater }\end{array}$ & 10.10 .1929 & $\begin{array}{l}\text { The Land of } \\
\text { Smiles }\end{array}$ & Drury Lane & 8.5 .1931 \\
\hline $\begin{array}{l}\text { Im Weißen } \\
\text { Rössl }\end{array}$ & $\begin{array}{l}\text { Grosses Schau- } \\
\text { spielhaus }\end{array}$ & 8.11 .1930 & $\begin{array}{l}\text { White Horse } \\
\text { Inn }\end{array}$ & $\begin{array}{l}\text { London } \\
\text { Coliseum }\end{array}$ & 8.4 .1931 \\
\hline Die Dubarry & Admiralspalast & 14.8 .1931 & The Dubarry & $\begin{array}{l}\text { His Majesty's } \\
\text { Theatre }\end{array}$ & 14.4 .1932 \\
\hline $\begin{array}{l}\text { Eine Frau, die } \\
\text { weiß, was sie } \\
\text { will }\end{array}$ & $\begin{array}{l}\text { Metropol- } \\
\text { Theater }\end{array}$ & 1.9.1932 & $\begin{array}{l}\text { Mother of } \\
\text { Pearl }\end{array}$ & Gaiety Theatre & 27.1 .1933 \\
\hline Ball im Savoy & $\begin{array}{l}\text { Metropol- } \\
\text { Theater }\end{array}$ & 23.12 .1932 & $\begin{array}{l}\text { Ball at the } \\
\text { Savoy }\end{array}$ & Drury Lane & 8.9.1933 \\
\hline
\end{tabular}


Tabelle 9: Anzahl der vom Lord Chamberlain genehmigten und verbotenen Stücke, 1860-1912

\begin{tabular}{|c|c|c|}
\hline Jahre & Genehmigung & Verbote \\
\hline 1860 & 242 & 3 \\
\hline 1861 & 217 & 1 \\
\hline 1862 & 201 & 1 \\
\hline 1863 & 220 & 0 \\
\hline 1864 & 200 & 0 \\
\hline 1865 & 181 & 1 \\
\hline 1866 & 173 & 0 \\
\hline 1867 & 174 & 0 \\
\hline 1868 & 159 & 0 \\
\hline 1869 & 150 & 2 \\
\hline 1870 & 184 & 2 \\
\hline 1871 & 263 & 5 \\
\hline 1872 & 206 & 1 \\
\hline 1873 & 247 & 0 \\
\hline 1874 & 178 & 1 \\
\hline 1875 & 213 & 0 \\
\hline 1876 & 344 & 0 \\
\hline 1877 & 272 & 4 \\
\hline 1878 & 204 & 0 \\
\hline 1879 & 272 & 0 \\
\hline 1880 & 252 & 0 \\
\hline 1881 & 286 & 0 \\
\hline 1882 & 302 & 0 \\
\hline 1883 & 287 & 1 \\
\hline 1884 & 320 & 0 \\
\hline 1885 & 288 & 2 \\
\hline 1886 & 294 & 3 \\
\hline 1887 & 304 & 3 \\
\hline 1888 & 348 & 0 \\
\hline 1889 & 287 & 1 \\
\hline 1890 & 297 & 0 \\
\hline 1891 & 244 & 1 \\
\hline 1892 & 396 & 1 \\
\hline 1893 & 362 & 0 \\
\hline 1894 & 433 & 0 \\
\hline 1895 & 374 & 4 \\
\hline 1896 & 461 & 2 \\
\hline 1897 & 481 & 3 \\
\hline 1898 & 440 & 2 \\
\hline 1899 & 464 & 6 \\
\hline 1900 & 466 & 3 \\
\hline 1901 & 513 & 2 \\
\hline 1902 & 519 & 2 \\
\hline 1903 & 538 & 3 \\
\hline 1904 & 468 & 1 \\
\hline 1905 & 520 & 2 \\
\hline 1906 & 579 & 2 \\
\hline 1907 & 536 & 4 \\
\hline 1908 & 560 & 4 \\
\hline 1909 & 577 & 3 \\
\hline 1910 & 604 & 2 \\
\hline 1911 & 608 & 6 \\
\hline 1912 & 1070 & 6 \\
\hline
\end{tabular}

Quelle: Fowell und PALMER, Censorship in England, S. 353. 
432 Tabellen

Tabelle 10: Anzahl der in Berlin genehmigten und verbotenen Stücke, 1876-1900

\begin{tabular}{lrrrrr}
\hline Jahr & genehmigt & neu eingereicht & verboten & neu eingereicht & Insgesamt \\
\hline 1876 & & & 7 & & 231 \\
1877 & & 8 & & 210 \\
1878 & & 11 & & 218 \\
1879 & & 25 & & 1284 \\
1880 & & 18 & & 719 \\
& & & & \\
1891 & & 10 & 219 & 419 \\
1892 & 98 & 111 & 9 & 236 & 468 \\
1893 & 106 & 121 & 12 & 231 & 577 \\
1894 & 115 & 104 & 15 & 208 & 678 \\
1895 & 100 & 93 & 17 & 243 & 1208 \\
1896 & 119 & 109 & 27 & 297 & 1105 \\
1897 & 126 & 104 & 22 & 421 & 1486 \\
1898 & 134 & 136 & 11 & 336 & 1969 \\
1899 & 217 & 182 & 19 & 294 & 1051 \\
1900 & 174 & 151 & 82 & & \\
\hline
\end{tabular}

Quelle: Verwaltungs-Bericht, S. 63; DRITTER Verwaltungsbericht, S. 373-374. 
Tabelle 11: Schauspielerinnen und Schauspieler in London und Großbritannien

\begin{tabular}{lrrr}
\hline Großbritannien & \multicolumn{3}{c}{} \\
\hline Jahr & Frauen & Männer & Insgesamt \\
1861 & 1139 & 1311 & 2450 \\
1871 & 1093 & 1809 & 2902 \\
1881 & 2368 & 2197 & 4565 \\
1891 & 3696 & 3625 & 7321 \\
1901 & 6443 & 6044 & 12487 \\
1911 & 9171 & 9076 & 18247 \\
1921 & & & \\
1931 & 9620 & 8210 & 17830 \\
\hline
\end{tabular}

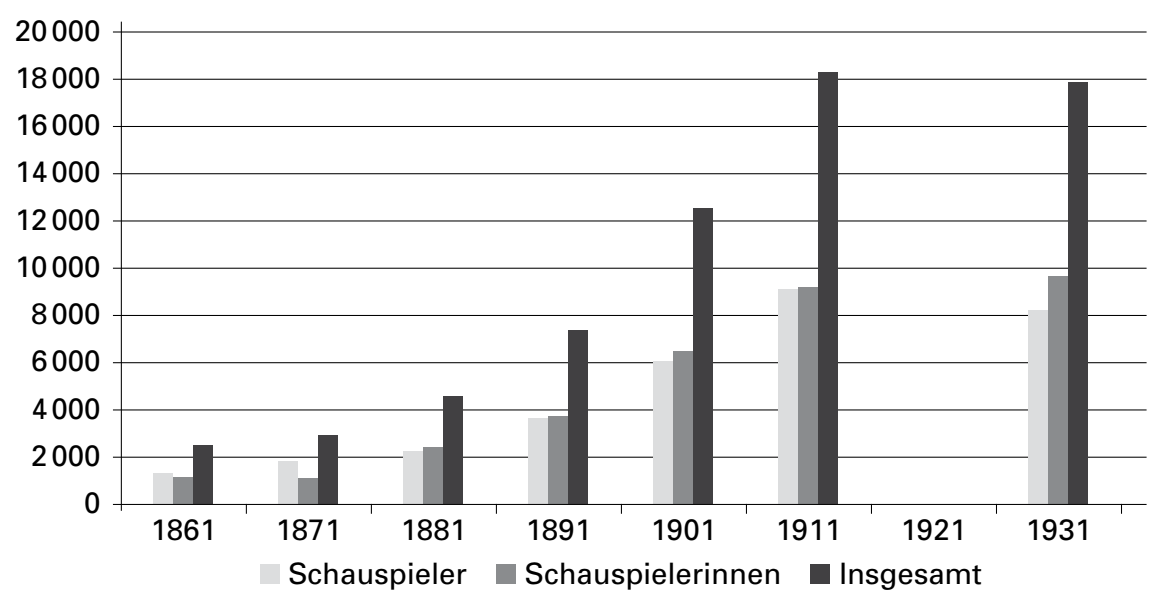




\begin{tabular}{lrrr}
\hline London & \multicolumn{3}{c}{} \\
\hline Jahr & Frauen & Männer & Insgesamt \\
1861 & 400 & 493 & 893 \\
1871 & 782 & 723 & 1505 \\
1881 & 1090 & 788 & 1878 \\
1891 & 1664 & 1271 & 2935 \\
1901 & 2911 & 2234 & 5145 \\
1911 & 4057 & 3338 & 7395 \\
1921 & 3860 & 3311 & 7171 \\
1931 & 3918 & 3485 & 7403 \\
\hline
\end{tabular}

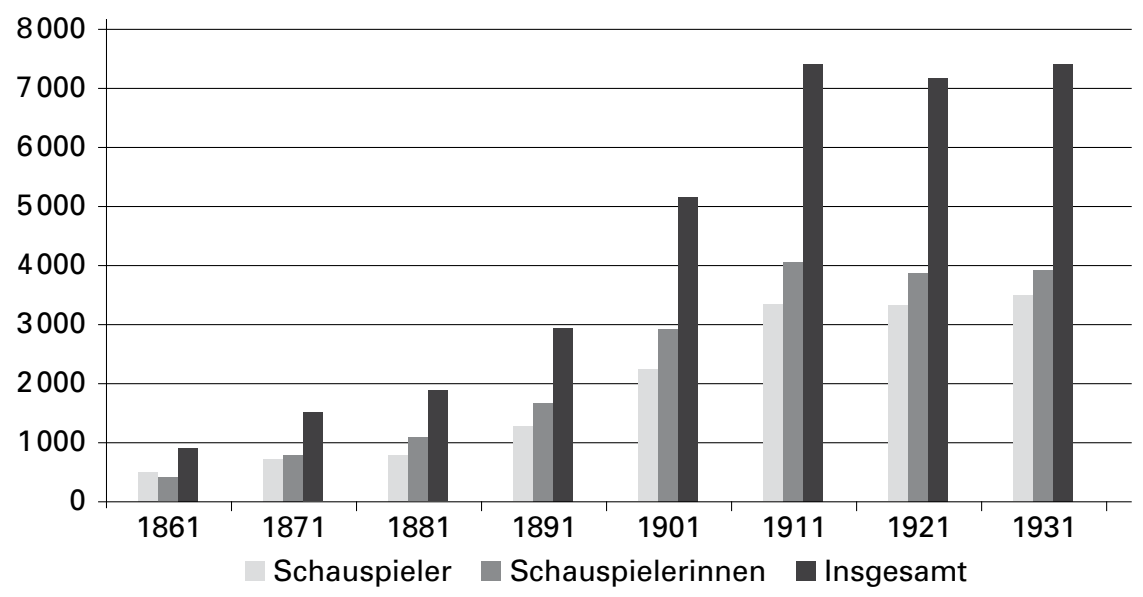

Quelle: Census of England and Wales for the year 1861, 2. Bd.; Census of ENGLand and Wales 1871, 3. Bd.; Census of England and Wales 1881, 3. Bd.; Census of England and Wales 1891, 3. Bd.; Census of ENGland and Wales 1901; Census of England and Wales 1911, 10. Bd.; Census of England and Wales 1921; Census of ENGLAND AND WaLES 1931. 
Tabelle 12: Angestellte im Bereich Musik und Theater

\begin{tabular}{lrrr}
\hline Deutschland & \multicolumn{3}{c}{} \\
\hline Jahr & Frauen & \multicolumn{3}{c}{ Männer } & Insgesamt \\
1882 & 6081 & 40427 & 46508 \\
1895 & 10369 & 55196 & 65565 \\
1907 & 22902 & 90699 & 90699 \\
1925 & 27439 & 53432 & 80871 \\
\hline
\end{tabular}

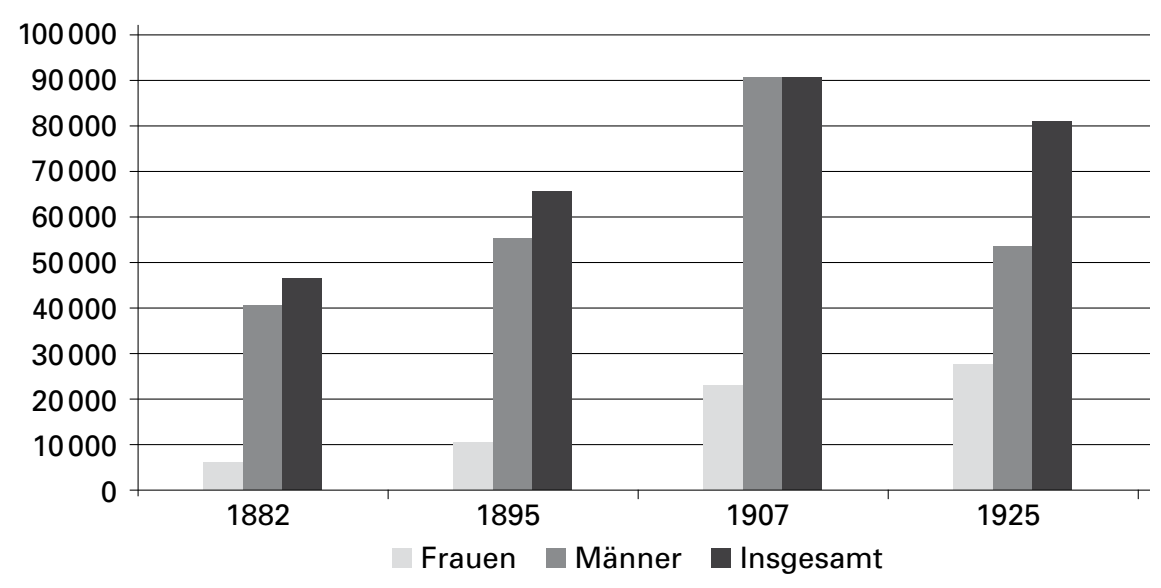

\begin{tabular}{lrrr}
\hline Berlin & \multicolumn{3}{c}{} \\
\hline Jahr & Frauen & Männer & Insgesamt \\
1882 & 812 & 2598 & 3410 \\
1895 & 1667 & 4293 & 5960 \\
1907 & 2862 & 6179 & 9041 \\
1925 & 5982 & 7508 & 13446 \\
\hline
\end{tabular}

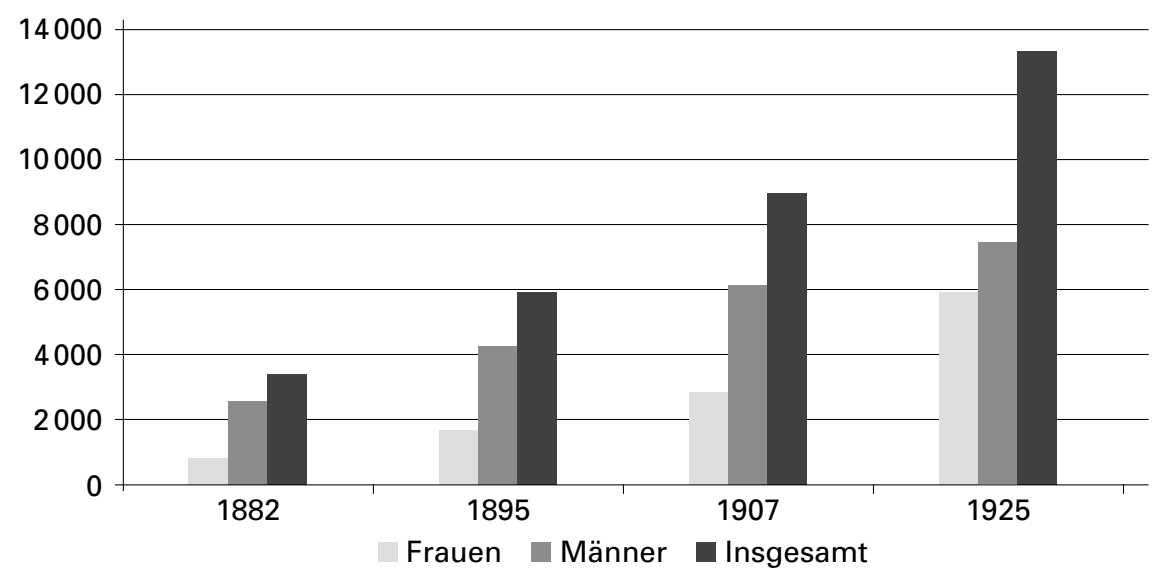

Quelle: Statistik des Deutschen Reichs, Neue Folge, Bd.211, Abteilung X, S. 98, 102, 236; Statistik DeS Deutschen Reiches, Bd. 402,I, Teil I, S. 394-395; Statistik des Deutschen Reiches, Bd. 406,1, Heft 1, S. 16. 